
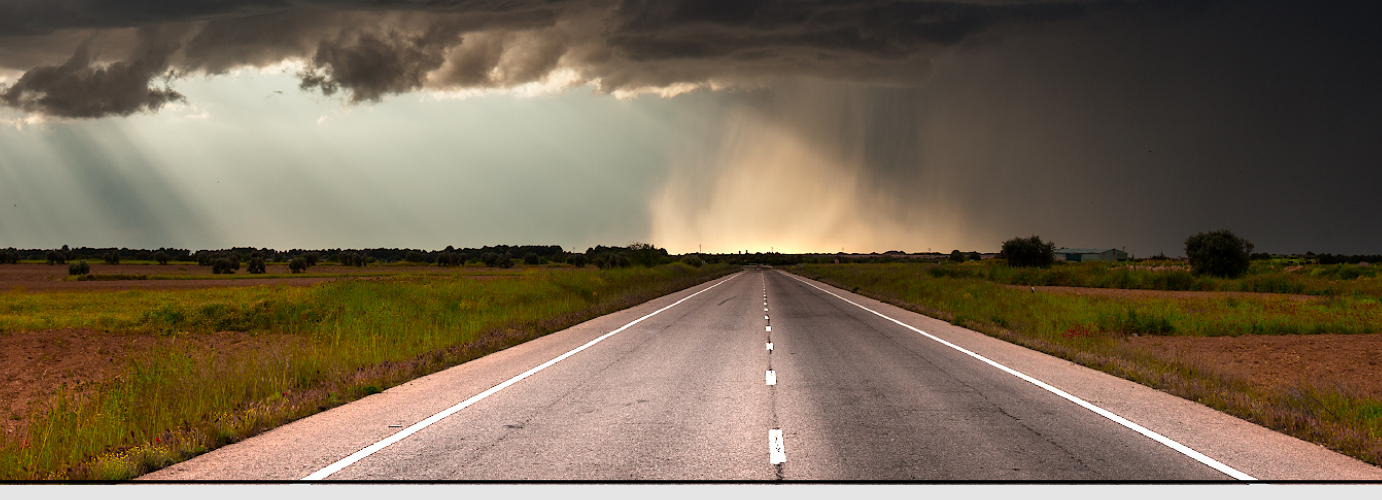

\title{
Proyecto PROFORCE
}

Clemens Wastl, Yong Wang, Martin Kulmer and Andrea Sigl

Department of Forecasting Models, Zentralanstalt für Meteorologie und Geodynamik (ZAMG), Austria

ANDRE SIMON

Hungarian Meteorological Service (OMSZ), Hungary

TRAD. FranCisco MARTÍN LEÓN

Meteorólogo

Ex Meteorólogo del Estado, Agencia Estatal de Meteorología (AEMET)

Coordinador de la Revista gratuita del Aficionado a la Meteorología

(RAM https: //www. tiempo. com/ram/)

Se presenta un proyecto multidisciplinar llevado a cabo en Austria y Hungría de colaboración entre meteorólogos y personal de protección civil de ambos países donde la predicción probabilista «sin costuras» $\mathrm{o}$ «sin discontinuidades» (seamless) ocupa un lugar muy destacado a la hora de predecir fenómenos severos. Este capítulo está basado en un informe técnico de la Organización Meteorológica Mundial (OMM) [1].

Palabras clave: fenómenos meteorológicos adversos, FMA, fenómenos meteorológicos extremos, plan Meteoalerta, avisos meteorológicos, proyecto PROFORCE, sistemas de predicción por conjuntos aplicados a la emisión de avisos meteorológicos.

Imagen parte superior: base de un cumulonimbus. Yepes (Toledo), hacia el noroeste, 14 de mayo de 2013, a las 18:52. Fotografía de José Antonio Quirantes CALVo. 


\subsection{Proyecto PROFORCE}

La frecuencia y la intensidad de fenómenos meteorológicos extremos están aumentando debido al cambio climático. La falta de preparación para tales fenómenos extremos puede dar lugar a daños económicos significativos y pérdida de vidas. Por lo tanto, los pronósticos meteorológicos precisos y fiables son esenciales para que las agencias de protección civil se preparen mejor y para las poblaciones en riesgo.

El proyecto Bridging of Probabilistic Forecasts and Civil Protection, (Tendiendo un puente entre la predicción probabilista y la protección civil) conocido abreviadamente como PROFORCE [1] http: //www.echo-proforce.eu/, dio un primer paso en esta dirección, proporcionando información significativa para la toma de decisiones, basada en el impacto a las autoridades civiles responsables de la protección de los ciudadanos, el medio ambiente y la propiedad.

Las previsiones meteorológicas están sujetas a incertidumbres inherentes debido a la naturaleza caótica de la atmósfera y a las limitaciones de los modelos de predicción meteorológica. Los sistema(s) de predicción por conjuntos (SPC) son ampliamente utilizados por la comunidad meteorológica para cuantificar estas incertidumbres, pero éstas no se comunicaron a los usuarios finales hasta hace poco. Sin embargo, esa información sobre las incertidumbres tiene un gran potencial para mejorar los procesos de toma de decisiones de tiempo crítico en las agencias de protección civil y otras partes interesadas, especialmente si están adaptadas a sus necesidades y redactadas con su terminología. PROFORCE ha logrado esto gracias a la colaboración multidisciplinaria e intersectorial entre los servicios meteorológicos y los usuarios finales. Ha aumentado la conciencia de los impactos potenciales de eventos extremos en la protección civil.

\subsection{Un sistema de pronóstico pro- babilista sin costuras o sin dis- continuidad}

PROFORCE comenzó en diciembre de 2013 y duró dos años. Fue cofinanciado por el Servicio de Ayuda Humanitaria y Protección Civil de la Comisión Europea (ECHO) y dirigido por el Servicio Meteorológico de Austria, ZAMG. Basándose en la cooperación transnacional entre los servicios meteorológicos nacionales austriacos y húngaros (ZAMG y OMSZ, respectivamente) y los socios de protección civil en los distritos de Baja Austria y el condado de Somogy, PROFORCE tenía como objetivo mejorar los procedimientos de preparación y toma de decisiones en las agencias de protección civil.

Sistema de pronóstico probabilista innovador. La característica principal del sistema de previsión sin fisuras y continuo de PROFORCE es su carácter probabilista que contiene información sobre la incertidumbre y la previsibilidad (o predecibilidad, cap. 12 en la página 155) de fenómenos meteorológicos severos. Los organismos de protección civil deberían poder utilizarlo para optimizar su proceso de toma de decisiones en términos de preparación y concienciación y, por lo tanto, proteger mejor a la sociedad y al medio ambiente frente al impacto del tiempo severo. El pronóstico probabilista sin fisuras o sin costuras de PROFORCE combina cuatro sistemas diferentes:

- El sistema de predicción probabilista EPS del Centro Europeo de Pronósticos del Medio Plazo (ECMWF/CEPMP), que representa la predicción del tiempo a medio plazo y a nivel sinóptico;

- El Sistema de predicción probabilista EPS de Área Limitada de Europa Central (ALADIN-LAEF), que representa el tiempo a nivel regional (ALADINHUNEPS para el dominio húngaro)

- El EPS del modelo de mesoescala francés (ARO$\mathrm{ME}$ ), que proporciona pronósticos hasta 30 horas de anticipación con un enfoque en la convección; y

- El Conjunto de Nowcasting Integrado con Análisis Exhaustivo (Integrated Nowcasting through Comprehensive Analysis (INCA) Ensemble), que proporciona información sobre la toma de decisiones para ayudar a las agencias de protección civil a hacer mejores juicios ante los riesgos de desastre inminentes.

Cada sistema juega su propio papel en el producto final sin costura según la naturaleza del evento meteorológico previsto (ya sea convectivo o a gran escala) y el rango de pronóstico. Bajo la suposición de que cada EPS produce los mejores resultados disponibles para su marco de tiempo designado, cada paso de tiempo individual en el pronóstico utiliza los datos del EPS correspondiente, haciendo así que el pronóstico sea uniforme. Así, cada día que un evento extremo se acerca, la precisión de las previsiones y advertencias mejoran.

Los distintos vacíos en los pasos de tiempo, que resultan de los diferentes modelos de referencia, se reflejan 
en el producto final, en la medida que el proyecto PROFORCE capacita y entrena específicamente al personal de protección civil para manejar tales incertidumbres.

Advertencias sin fisuras, acciones igualmente sin fisuras. El intercambio sin fisuras de información y las acciones entre los meteorólogos y el personal de protección civil siguen esta estructura:

Preavisos / respuesta a medio plazo. El SPC del ECMWF (ECENS, sec. 19.3 en la página 293) muestra un posible fenómeno meteorológico severo con una probabilidad relativamente alta y se emite un primer aviso previo a las agencias de protección civil, que entran en la fase de acción de «respuesta» y toman medidas tempranas e iniciales, como organizar y orientar los turnos.

Avisos más específicos / preparación. Dos o tres días antes del evento potencial, el siguiente SPC en la cadena hace pronósticos, genera y provee productos y avisos más precisos tanto en cuanto al espacio como al tiempo. Por lo tanto, puede dar una advertencia más específica. Sobre esta base, el personal de protección civil entra en la fase de «preparación» con actividades intensificadas como la provisión de equipo y personal. Un día después de esto, el EPS que permite la convección proporciona más detalles, particularmente en la topografía tan compleja como las regiones en los Alpes.

Nowcasting y entrar en acción. Por último, el material EPS de alcance inmediato proporciona la imagen más precisa de la situación meteorológica severa y se pueden tomar decisiones finales. El personal de protección civil entra en la tercera fase de «acción en marcha» en la que se ponen en marcha planes finales, como concentrar las actividades operacionales en las regiones más afectadas. Este enfoque de predicción inmediata es especialmente importante para los eventos convectivos de la temporada de verano que se caracterizan por una alta variabilidad espacial y temporal. Por esta razón, el modelo INCA Ensemble se ejecuta cada hora mientras que otros modelos -
AROME EPS, ALADIN-LAEF y ECENS - solo se actualizan cada 12 horas; en el caso de Hungría, el modelo ALADIN-HUNEPS sólo se actualiza una vez cada 24 horas.

\subsection{Creación de portales web}

Los meteorólogos y el personal de protección civil trabajaron juntos para desarrollar conjuntos apropiados de datos meteorológicos que ilustrasen las previsiones de manera clara y sencilla, promoviendo así un rápido proceso de toma de decisiones. En primer lugar, los socios de las protecciones civiles definieron umbrales razonables para los parámetros meteorológicos clave: velocidad del viento, precipitaciones y temperatura.

Estos umbrales diferían considerablemente en los dos países. Por ejemplo, una ráfaga de viento de $60 \mathrm{~km} / \mathrm{h}$ es normal y ocurre con frecuencia en las regiones montañosas de Austria, pero representa un serio peligro para la extremadamente vulnerable región del Lago Balatón en Hungría, con muchas actividades de deportes acuáticos en verano. Como resultado, se establecieron dos portales web independientes, uno para cada país con diferentes umbrales y visualizaciones.

En los sitios web, las ilustraciones de los productos se preparan de tal manera que el pronóstico parece ser transparente, es decir, las fuentes de mediana y menor resolución se reemplazan automáticamente por pronósticos de mayor resolución en rangos más cortos. Por lo tanto, los usuarios no necesitan identificar el SPC en cuestión que se está utilizando. La salida de los sistemas individuales de SPC se visualiza, tanto como mapas de probabilidad, que muestran la probabilidad de superar ciertos umbrales, como información de puntos para ubicaciones preseleccionadas, en forma de meteogramas o penachos.

Los mapas de probabilidad en el portal austriaco (ejemplo en Figura 42.1) están acompañados por elementos de imagen que ilustran el nivel de amenaza de una manera similar a las señales de semáforo: verde, amarillo, naranja y rojo a medida que aumenta la amenaza. 


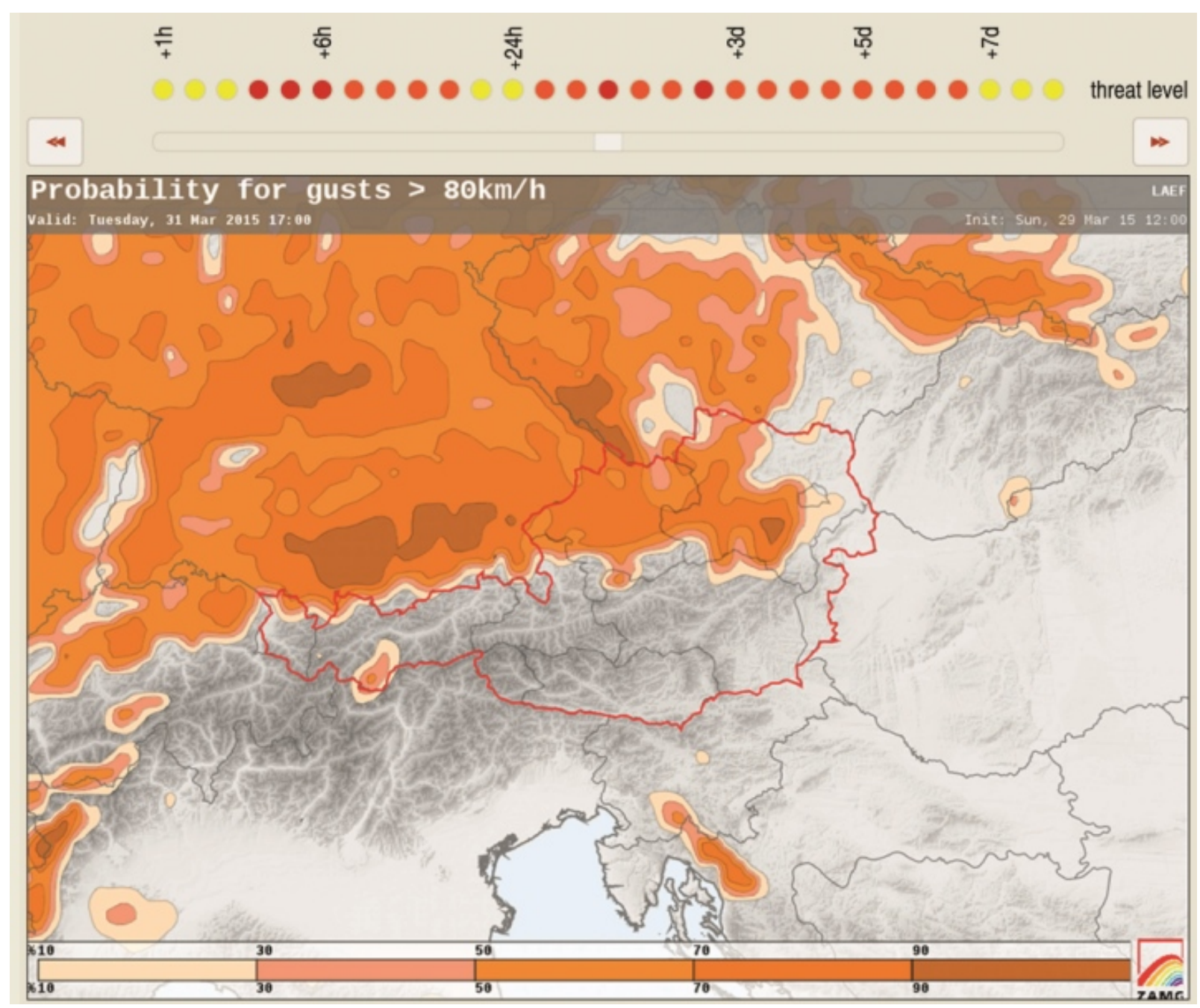

Figura 42.1: La información de pronóstico probabilista sin costura se procesa, visualiza y luego se publica en un portal web construido específicamente, al que pueden acceder las autoridades de protección civil y los gestores de desastres (ejemplo del portal web austríaco).

Se creó un índice global de avisos (Figura 42.2) para proporcionar a las autoridades de protección civil información general inmediatamente después de entrar al portal. Tiene en cuenta la probabilidad de un evento, su grado de adversidad (intensidad) y el momento en el que se espera, dado que un pronóstico a más largo plazo generalmente contiene mayores incertidumbres. En otras palabras, un aviso «amarillo» con una semana de antelación no debería causar pánico, pero un aviso «rojo» en la previsión del día siguiente debería sonar con campanas de alarma.

\subsection{Formación y experiencia de primera mano}

La cooperación interdisciplinaria entre meteorólogos y organismos de protección civil fue clave para el éxito de PROFORCE. El personal de protección civil tuvo que familiarizarse con las previsiones probabilistas para obtener el máximo beneficio del portal web. Las sesiones de formación en ambos países ayudaron a fortalecer la cooperación transnacional y la retroalimentación de las agencias de protección civil sobre la información probabilista que también permitió a los desarrolladores de modelos mejorar sus SPC.

Se realizaron pruebas intensas del sistema durante los eventos meteorológicos severos en las regiones piloto de la Baja Austria y el Condado de Somogy durante el transcurso del proyecto (diciembre de 2013 a noviembre de 2015). La retroalimentación de los usuarios finales y los beneficiarios fue en general positiva y se confirmó la aplicabilidad del sistema para el uso operativo en las agencias de protección civil. Las acciones de prevención y preparación podrían iniciarse mucho antes y ser más específicas con el nuevo sistema que con el pronóstico meteorológico clásico determinista. 


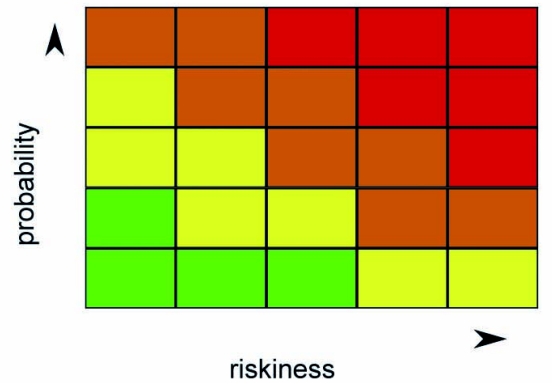

riskiness

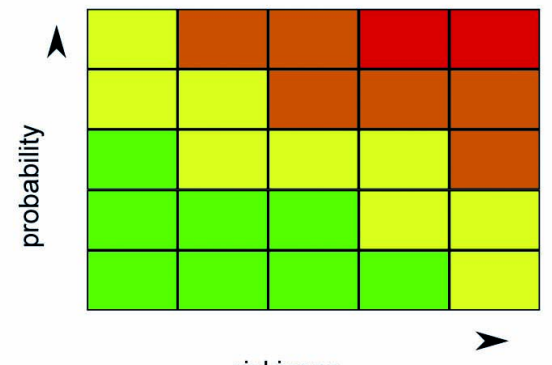

riskiness

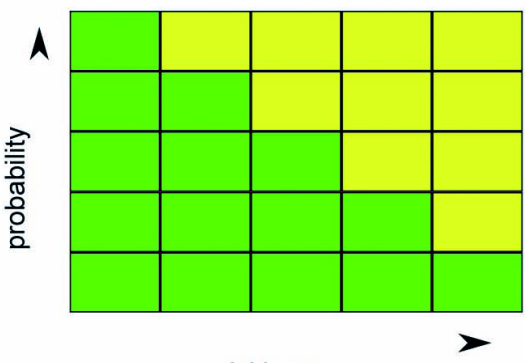

riskiness

time

Figura 42.2: Combinación de riesgo, probabilidad y plazo de predicción para establecer un nivel de amenaza común.

La borrasca intensa NikLAS ( 30 de marzo - 2 de abril de 2015) ofreció un buen ejemplo de ello. Las probabilidades altas de rachas de viento que excedían los umbrales en amplias zonas de Austria fueron señaladas por el ECENS con siete días de anticipación. Una vez que un patrón de tiempo severo aparece por primera vez en el ECENS global, es necesario comprobar continuamente la consistencia de su posición, tiempo e intensidad de un pronóstico al siguiente. Esto condujo al anuncio de preadvertencias iniciales en las regiones relevantes cinco días antes del inicio del evento. Tres días antes del evento, el EPS de mayor resolución mostró probabilidades muy altas de ráfagas por encima de $80 \mathrm{~km} / \mathrm{h}$ (nivel 2) y altas probabilidades de ráfagas superiores a $100 \mathrm{~km} / \mathrm{h}$ (nivel 3, el nivel de advertencia más alto). Al final, la racha máxima registrada en las estaciones meteorológicas de baja altitud fue de $121 \mathrm{~km} / \mathrm{h}$ en Enns, Alta Austria. La información adicional sobre la fiabilidad y la incertidumbre del nuevo sistema facilitó una mejor evaluación de la situación; sin embargo, el EPS de nowcasting no proporcionó beneficio adicional.

El rendimiento se comprobó verificando los avisos contra los mapas de despliegue de la agencia de protección civil. Hubo una correlación muy alta entre la densidad de las acciones de protección civil y las áreas resaltadas en los pronósticos del EPS, especialmente para los temporales a gran escala y las inundaciones.

En el centro de gestión de desastres húngaro, el sistema de pronóstico probabilista se utilizó ampliamente en los preparativos para varios concursos en y alrededor del lago Balaton durante las temporadas de verano de 2014 y 2015, incluyendo la Blue Ribbon Race y el Lake Balaton Cross-Swimming. Para el último evento, la información de EPS fue útil cuando se trató de decidir si se pospondría o no el concurso. Las actividades en el lago Balaton son vulnerables a los cambios repentinos de tiempo, que a veces puedes estar confinados regionalmente. La experiencia con PROFORCE demostró que si bien las técnicas de pronóstico de EPS pueden mejorar la previsibilidad de estos cambios, sigue siendo necesario un mayor desarrollo, particularmente en el rango de predicción a muy corto plazo.

\subsection{Continuar en el futuro}

PROFORCE fue un proyecto desafiante que esencialmente tenía dos objetivos:

El primero fue construir un puente entre la comunidad de predicción, que monitorea el tiempo y conoce las fortalezas y debilidades de cada modelo individual, y la comunidad de protección civil, que se enfoca en los impactos del tiempo severo. El entendimiento efectivo entre estos dos grupos era un desafío.

El segundo objetivo se refería al manejo de las probabilidades meteorológicas. Con diferentes grados de familiaridad con la investigación meteorológica y climática, tanto las autoridades de protección civil como los predictores del tiempo están empezando a aprender a manejar y confiar en las probabilidades. El personal de protección civil debe recibir una formación regular para que puedan confiar más en la aplicación del concepto de pronóstico probabilista cuando tomen decisiones cotidianas sobre el despliegue de ayudas o la cancelación de eventos de masas.

Es importante destacar que un SPC sin costuras no reemplaza completamente los avisos estándar emitidos por la oficina de pronóstico, que también tiene en cuenta resultados de otros modelos de predicción numérica y las observaciones (Figura 42.3). Pretende, 
sobre todo, proporcionar información y detalles adicionales sobre la distribución espacial y la intensidad de los fenómenos meteorológicos.

En última instancia, todo el mundo en la cadena de avisos y alertas, desde las autoridades oficiales hasta los interesados locales y finalmente el público en ge- neral, puede beneficiarse de este proyecto. El tiempo cambiante siempre presenta nuevos desafíos para los meteorólogos y expertos en protección civil. Aunque el proyecto PROFORCE ya ha finalizado, la cooperación transregional e interdisciplinaria que estableció continuará en el futuro.
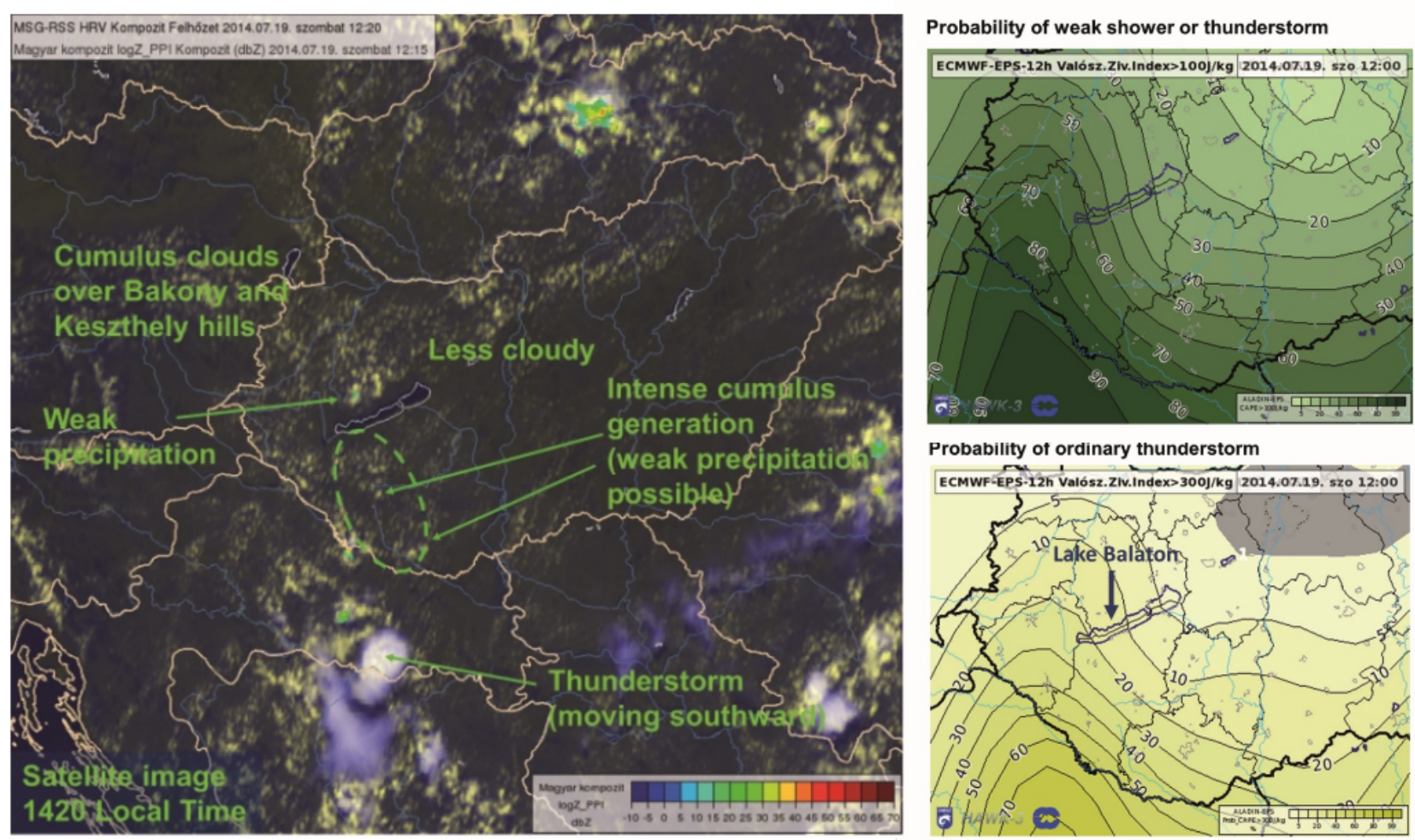

Figura 42.3: Izquierda: imagen Meteosat (MSG) compuesta por HRV (visible alta resolución) e imagen infrarroja que muestra la situación durante la competición Balaton Cross-Natación el 19 de julio de 2014. Derecha: Pronóstico de probabilidad del ECENS del índice de tormentas (CAPE) superior a $100 \mathrm{~J} / \mathrm{kg}$ (arriba) y superior a $300 \mathrm{~J} / \mathrm{kg}$ (abajo). 


\subsection{Referencias}

[1] Wastl, Clemens y col. Probabilistic Forecasts and Civil Protection. Informe téc- nico 66. 2017. URL: https : / / public . wmo . int / en / resources / bulletin / probabilistic - forecasts - and civil-protection (citado en páginas 633, 634). 